\title{
Hypoxia induced metastasis of gastric cancer through HIF1a/ANXA1/MMP-2 pathway
}

Qing-Hua Liu ( $D$ liuqinghua1@126.com )

Xuzhou Medical University https://orcid.org/0000-0001-5941-453X

Lei Xia

Xuzhou Medical University

Chen Chen Lu

Xuzhou Medical University

Ying Liu

Xuzhou Medical University

Primary research

Keywords: HIF1a, ANXA1, MMP-2, gastric cancer, metastasis

Posted Date: June 16th, 2020

DOI: https://doi.org/10.21203/rs.3.rs-35116/v1

License: (c) (i) This work is licensed under a Creative Commons Attribution 4.0 International License.

Read Full License 


\section{Abstract}

\section{Background}

The aim of this study was to investigate the mechanism of hypoxia induced metastasis of gastric cancer (GC). Method

This study explored the clinical significance of hypoxia inducible factor (HIF) 1a and annexin A1 (ANXA1) in human GC by immunohistochemistry of a tissue microarray with 76 surgically resected GC specimens and further examined the mechanism of the role of HIF1a in GC.

Results

Our data demonstrated that HIF1a expression was dramatically increased in GC tissues. High HIF1a expressions were significantly correlated with lymph node metastasis and with worse survival in GC patients. In addition, HIF1a expression was an independent prognostic factor in GC patients and the combination of HIF1a and ANXA1 might serve as useful prognostic markers in GC patients. Furthermore, HIF-1a could promote GC migration and invasion through HIF1a/ANXA1/MMP-2 (Matrix metal proteinase 2) pathway.

\section{Conclusions}

Our findings indicated that HIF-1a may serve as a promising prognostic biomarker and therapeutic target for inhibiting GC metastasis.

\section{Background}

GC is the fifth malignancy tumor worldwide [1], the second in China [2]. Furthermore, it's the third leading cause of cancer related mortalities worldwide, the second in China. Most GC patients are diagnosed at advanced stage which results in a low 5-year survival rate, in spite of the rapid improvements in surgery and chemotherapy during recent years [1,3]. Metastasis is the main reason for the high mortality of GC and it is poorly understood $[4,5]$. The process of tumor metastasis was regulated by the genetic changes of tumor cells and the tumor microenviroment (TME) $[6,7]$.

Hypoxia emerges as a prominent feature of the TME, stimulates protective cellular response through changing specific genes of tumor cells, then, regulating cell growth, apoptosis, cell survival, angiogenesis, cell invasion and migration [8]. Tumors grown in hypoxic TME are more likely to metastasis and prone to chemoresistance [9]. One of the important regulators of this process is HIF, it is necessary for tumor metastasis and its downstream proteins may play important roles in tumor metastatic progression [10]. Biochemically, HIF is consisted of one of HIF-1a, HIF-2a, HIF-3a subunits binding to HIF-1 $\beta$. Among the heterodimers of HIFs, HIF-1a is widely studied in the growth process of tumor, which is the key of transcription factors and the physiological regulators. 
Loss of HIF-1a could sustain tumor metabolism and cellular proliferation by upregulating ANXA1 and they expressed in a mutual exclusive manner to in 37 cell lines of GC [11]. ANXA1 is one of the important proteins of the downstream pathways under hypoxia environment regulated by HIF1 [11]. As an important marker and an attractive therapeutic target of malignant tumor, ANXA1 is a 37-kDa protein belonging to the annexin superfamily, besides its multiple physiological functions, recent evidences implicated ANXA1 strongly affects migration and invasion of tumor cells [12-14].

In this study, we aimed to investigate whether hypoxia could regulate metastasis of gastric cancer through HIF1a/ANXA1 pathway. We firstly investigated the expressions of HIF-1a and ANXA1 in GC tissues and analyzed the clinicopathological and prognostic significance of HIF-1a and ANXA1 in GC patients. Furthermore, we found that hypoxia could improve migration and invasion of GC cells through HIF-1a/ANXA1/MMP-2 pathway. Our results indicated that HIF-1a may act as diagnostic and prognostic marker and may be a potential therapeutic target for GC.

\section{Materials And Methods}

\section{Patients and specimens}

Formalin fixed paraffin-embedded GC tissues and the adjacent tissues (control) used for immunohistochemistry were obtained from 76 GC patients undergoing gastrectomy in the Affiliated Hospital, Xuzhou Medical University, China, 2009. The patients were comprised of 56 males and 20 females, aged from 23 to 85 years (Table 1,2). All of these patients had accepted no chemotherapy in prior to their gastrectomy surgeries. Until May, 2015, 14 of 76 patients were still alive (the survival period until May 2015 was 62 months). Whereas 39 of 76 patients were dead, and there were 23 patients lost contacted during the following-up.

Table 1

Expression of HIF-1a and ANXA1 in GC

\begin{tabular}{|c|c|c|c|c|c|c|}
\hline & \multicolumn{2}{|l|}{ HIF-1a } & \multirow[t]{2}{*}{$P$} & \multicolumn{2}{|l|}{ ANXA1 } & \multirow[t]{2}{*}{$P$} \\
\hline & $+(\%)$ & $-(\%)$ & & $+(\%)$ & $-(\%)$ & \\
\hline None cancer & $22(32.8)$ & $45(67.2)$ & \multirow[t]{2}{*}{0.000} & $3(4.5)$ & $64(95.5)$ & \multirow[t]{2}{*}{0.000} \\
\hline Cancer & $63(82.9)$ & 13(17.1) & & $27(35.5)$ & $49(64.5)$ & \\
\hline
\end{tabular}


Table 2

Comparison of HIF-1a with Clinicopathological Features in GC patients

\begin{tabular}{|c|c|c|c|c|}
\hline \multirow[t]{2}{*}{ Variables } & \multicolumn{4}{|c|}{ HIF-1a Staining } \\
\hline & Positive(\%) & Negative(\%) & Total & $P$ \\
\hline \multicolumn{5}{|l|}{ Gender } \\
\hline Male & $45(80.4)$ & 11(19.6) & 56 & 0.329 \\
\hline Female & 18(90.0) & $2(10.0)$ & 20 & \\
\hline \multicolumn{5}{|l|}{ Age } \\
\hline$<60$ & $26(78.8)$ & $7(21.2)$ & 33 & 0.408 \\
\hline$\geq 60$ & $37(86.0)$ & $6(14.0)$ & 43 & \\
\hline \multicolumn{5}{|l|}{ TNM } \\
\hline$|-I|$ & $37(77.1)$ & 11(22.9) & 48 & 0.080 \\
\hline III-IV & $26(92.9)$ & $2(7.1)$ & 28 & \\
\hline \multicolumn{5}{|c|}{ Tumor Differentiation } \\
\hline Poor & $43(86.0)$ & $7(14.0)$ & 50 & 0.322 \\
\hline Moderate + Well & $20(76.9)$ & $6(23.1)$ & 26 & \\
\hline \multicolumn{5}{|l|}{ Tumor Diameter } \\
\hline$\leq 5 \mathrm{~cm}$ & $32(74.4)$ & $11(25.6)$ & 43 & 0.026 \\
\hline$>5 \mathrm{~cm}$ & $31(93.9)$ & $2(6.1)$ & 33 & \\
\hline \multicolumn{5}{|c|}{ Lymph Node Metastasis } \\
\hline Yes & $47(90.4)$ & $5(9.6)$ & 52 & 0.011 \\
\hline No & 16(66.7) & $8(33.3)$ & 24 & \\
\hline \multicolumn{5}{|c|}{ Hematogenous Metastasis } \\
\hline Yes & $9(100.0)$ & $0(0.0)$ & 9 & 0.149 \\
\hline No & $54(80.6)$ & 13(19.4) & 67 & \\
\hline
\end{tabular}

Frozen samples from 8 pairs of surgically resected GC tissues and their adjacent non-tumor tissues used for Western blot were obtained from GC patients undergoing gastrectomy in the Affiliated Hospital, Xuzhou Medical University, China, 2018. This study was approved by the Human Ethical Committee, the Institutional Review Boards of Affiliated Hospital of Xuzhou Medical University, and written informed consents were obtained from all patients. 
GC tissue microarray was constructed by a precision arraying instrument (Beecher Instruments, Silver Spring, MD, USA). The slides from GC tissue microarray were deparaffinized, rehydrated and heated in sodium citrate buffer $(0.01 \mathrm{~mol} / \mathrm{l}, \mathrm{PH} 6.0)$ for $8 \mathrm{~min}$ at $95^{\circ} \mathrm{C}$. Then, the slides were incubated with $3 \%$ hydrogen peroxide for $15 \mathrm{~min}$ at room temperature and blocked with normal goat serum (Beijing Sequoia Jinqiao Biological Technology Co., Ltd.). After drained off the blocking serum, the slides were labelled with two antibodies (rabbit anti-HIF-1 a monoclonal antibody: 20960-1-AP, Proteintech Group, Chicago, US; rabbit anti-ANXA1 polyclonal antibody: 21990-1-AP, Proteintech Group, Chicago, US) overnight at $4{ }^{\circ} \mathrm{C}$. The slides were rinsed for 10 min in PBS, 3 times and incubated for 30 min with the HRP-labeled polymer conjugated secondary antibody (12127A07, Beijing Sequoia Jinqiao Biological Technology Co., Ltd.). The dilution for both two antibodies was 1:400. The specific target(s) was visualized with 3, 3'diaminobenzidine (DAB) detection kit (Beijing Sequoia Jinqiao Biological Technology Co., Ltd.) and counterstained with hematoxylin.

Positive HIF-1 $a$ and ANXA1 staining appeared brown in cytoplasm with or without in nucleus. We graded positive staining according to both the stain intensity and the percentage of stained cells. Two pathologists independently examined all slides. The intensities of HIF-1 $a$ and ANXA1 staining were scored 0 to 3 ( $0=$ negative, $1=$ moderate; $3=$ strong). The percentage of stained cells was scored 1 to $4: 1$ ( $0-25 \%$ cells positively stained), $2(26-50 \%), 3(51-75 \%)$ and 4 (76-100\%). Final scores of both two proteins positive staining were evaluated by the immunoreactive score (IRS), which was calculated by multiplying the intensity score by the percentage score of positive cells. The negative staining of the specimens was IRS: 0.

Protein expressions were also evaluated by Western blotting. Equivalent amounts of total proteins from frozen samples of surgically resected GC tissues and their adjacent non-tumor tissues were extracted as according to the extraction protocol of the total protein kit, KeyGen Biotech, China). The concentration determined using BCA protein kit, Beyotime Biotechnology, Shanghai, China. The procedure of western blot analysis was performed as described previously [15]. Protein samples were denatured, electrophoresed in SDS/polyacrylamide gels and transferred into polyvinylidene difluoride membranes (Millipore). Antibodies against the following proteins were used: HIF-1a at a 1:2,000 dilution, ANXA1 at $1: 1,000$ and anti- $\beta$-actin at $1: 5,000$.

After incubation with secondary antibody: horseradish peroxidase-conjugated goat anti-rabbit IgG (ABL3012,AbSci[Washington[US) at a dilution of 1:10,000, protein bands were exposed to ECL system.

\section{Cell lines}

The human GC cell lines HGC27 and MGC803 were obtained from the Shanghai Institute of Biochemistry and Cell Biology, Chinese Academy of Science (Shanghai,

China). HGC27 cells were cultured in Roswell Park Memorial Institute 1640 medium (RPMI 1640, Gibco, California, US) supplemented with 10\% fetal calf serum (Hangzhou Sijiqing bioengineering Material Co., Ltd, Hangzhou, China); MGC803 cells were cultured in minimum essential medium (MEM, Gibco, 
California, US) supplemented with $10 \%$ fetal calf serum. These two cell lines were both incubated in a $37^{\circ} \mathrm{C}$ humidified incubator with $5 \% \mathrm{CO}_{2}$.

Hypoxia was achieved by exposing HGC27 and MGC803 cells in normoxic conditions with Cobalt chloride ( $\mathrm{CoCl}_{2}$, Sigma-Aldrich, St. Louis, MO, USA) $(300 \mu \mathrm{mol} / \mathrm{L})$ in their culture medium.

ANXA1 siRNA (5'-CCCGUUCUGAAAUUGACAUTT-3') and nonspecific control siRNA (5'-

UUCUCCGAACGUGUCACGUTT-3') were purchased from GenePharma (Suzhou, China). The control and ANXA1 siRNA were transfected into HGC27 and MGC803 cells with Lipofectamine 2000 reagent (Invitrogen, Shanghai, China) according to the manufacturer's instructions.

\section{Western blot}

Proteins from cells were extracted by the same procedure as described previously. The procedure of western blot was the same as above: extracted proteins from cells, antibodies of HIF-1a, ANXA1 and $\beta-$ actin. HIF-2a (1:2000, DF2928,Affinity Biosciences $\square$ Ohio[US), MMP-2 (1:1000, 10373-2-AP, Proteintech Group, Chicago, US), TIMP-2 (tissue inhibitor of metalloproteinase 2) (1:1000, 17353-1-AP, Proteintech Group), MMP-9 (Matrix metal proteinase 9) (1:1000, 10375-2-AP, Proteintech Group), TIMP-1 (tissue inhibitor of metalloproteinase 1) (1:1000, 10753-1-AP, Proteintech Group).

\section{Gelatin zymography}

Gelatin zymography was used to detect the activity of MMP-2. Cells $\left(2.5 \times 10^{6}\right)$ were seeded in $100-\mathrm{mm}$ plates and cultured for $3 \mathrm{~h}, 12 \mathrm{~h}$ and $24 \mathrm{~h}$. Then, proteins were collected and concentrated by Amicon Ultra-4-30 k centrifugal filters (Millipore, Billerica, MA, USA), $7500 \mathrm{~g}, 20 \mathrm{~min}$, at $4{ }^{\circ} \mathrm{C}$. We loaded $50 \mu \mathrm{g}$ of protein in non-denaturing conditions into $8 \%$ polyacrylamide gel containing $0.1 \%$ gelatin (China Pharmaceutical Shanghai chemical reagent station, Shanghai, China). After electrophoresis, gels were washed by $2.5 \%$ Triton $\mathrm{X}-100,30$ minutes. Then, gels were incubated for $48 \mathrm{~h}, 37^{\circ} \mathrm{C}$ in incubation buffer (50 mM Tris- $\mathrm{HCl}(\mathrm{pH} 8.8), 5 \mathrm{Mm} \mathrm{CaCl} 2,1 \mu \mathrm{M} \mathrm{ZnCl} 2$, and $0.02 \% \mathrm{NaN} 3)$, stained with Coomassie brilliant blue R-250 for 30 min (Coomassie Blue Staining Kit, Biotechnology Bioengineering Co., Ltd., Shanghai, China) following the steps provided by the manufacturer, and destained in destained solution for $15 \mathrm{~min}$, 3 times. The gelatinolytic activities were shown as clear areas in gel. Then, gels were photographed and quantitatively measured by scanning densitometry.

\section{Co-immunoprecipitation}

All operations are carried out at low temperature. The hypoxia model of gastric cancer cells HGC27 and MGC803 was established and the cells were harvested after $6 \mathrm{~h}$. Cells were lysed with IP RIPA on ice for 30 min.

The lysates were obtained by centrifuge at $12,000 \mathrm{rpm}$ for $15 \mathrm{~min}$ at $4{ }^{\circ} \mathrm{C}$ and the supernatant was the total protein. Add PBS to dilute the total protein to $1 \mu \mathrm{g} / \mu \mathrm{l}$, and incubate overnight with $1 \mu \mathrm{g} \mathrm{HIF-1a}$ antibody for every $500 \mu \mathrm{g}$ total protein. $30 \mu \mathrm{l}$ protein A/G agarose beads (SC-2003, Santa Cruz 
Biotechnology, California, US) were added to the cell lysate and incubated at $4{ }^{\circ} \mathrm{C}$ for $4 \mathrm{~h}$. The beads were washed 3 times in IP RIPA and subjected to Western blotting.

In order to detect the interaction between HIF-1 and ANXA1, and between HIF-1 and MMP-2, we incubated with antibodies of ANXA1 and MMP-2 respectively during the Western blotting.

\section{Immunofluorescence staining}

Identify the location of HIF-1a』ANXA1 and MMP-2 in GC cells by Immunofluorescence staining. Exponentially growing cells were seeded on glass slides in 12-well plates and were cultured in normoxic conditions to $\mathrm{CoCl} 2$ in their culture medium for $6 \mathrm{~h}$. The treated cells were rinsed three times with $0.01 \mathrm{M}$ PBS, and fixed for 20 min with $4 \%$ paraformaldehyde at $4{ }^{\circ} \mathrm{C}$. Afterwards, cells were permeabilized with $0.5 \%$ TritonX-100 for $15 \mathrm{~min}$. Later, cells were blocked with $10 \%$ normal goat serum for $1 \mathrm{~h}$. Primary antibodies: $100 \mu \mathrm{l} /$ well HIF-1a (1:300) and ANXA1 (1:300) mixture or MMP-2 (1:300) and ANXA1 (1:300) mixture were added and incubated at $4{ }^{\circ} \mathrm{C}$ overnight. After cells were rinsed with cold PBS three times, the secondary antibody was added and incubated at room temperature for $30 \mathrm{~min}$. Subsequently, the slides were stained with $100 \mu \mathrm{l} /$ well the corresponding Alexa Fluor 488 goat anti-rabbit IgG antibody (VA018, VICMED Life Sciences, Xu Zhou, China) and Alexa Fluor 594 goat anti-mouse IgG (VA019, VICMED Life Sciences, Xu Zhou, China) mixture at room temperature in dark for $1 \mathrm{~h}$. The nuclei were stained with $50 \mu \mathrm{l} /$ well 6-diamidino-2-phenylindole (DAPI) at room temperature in dark for $5 \mathrm{~min}$. Then, the slides were rinsed with PBS for 5 min, 3 times. Finally, the slides were analyzed by fluorescence microscope (Olympus BX-51, Olympus Corporation, Japan). All samples were processed in parallel.

\section{Wound healing assay}

Wound healing assay was used to detect the ability of cell migration. After transfecting HGC27 and MGC803 cells with ANXA1 siRNA and control siRNA, cells were grown to confluency, wound lines were made by scraping closed Pasteur pipette tips across the confluent cell layer. Then, cells were washed by PBS three times to remove detached cells and debris. The width of wound was observed and measured after $0 \otimes 6$ and 12 hours.

\section{Cell migration and invasion assays}

Cell migration and invasion assays were performed by using modified two chamber migration and invasion assays with a pore size of $8 \mu \mathrm{m}$. Transwell filters were inserted with or without Matrigel (BD Biosciences, USA) coating for invasion and migration assays, respectively. The detailed conditions have been described previously [16].

\section{Statistical analysis}

Data has been expressed as the means \pm SDs from at least three independent repeats. One-way analysis of variance (ANOVA) and multi-factorial ANOVA were used for comparing the differences between groups. Statistical analysis for the GC tissue microarray was performed by SPSS 16.0. The association between the staining of HIF-1a, ANXA1 and the clinicopathological parameters of GC patients, including gender, 
age, tumor size, TNM stage, lymph node and hematogenous metastasis were evaluated by the two-sided Fisher's exact test. The correlation between HIF-1a, ANXA1 expressions and patient survival was assessed by Kaplan- Meier and log-rank tests. Analysis of univariate and multivariate was analyzed by Cox regression model. Difference was considered significant when $P<0.05$.

\section{Results}

\section{The expression of HIF-1 aand ANXA1 in GC and non-tumor tissues}

We performed immunohistochemistry with the GC tissue microarray to study whether HIF-1a and ANXA1 expressions are changed in human GC tissues. According to immunohistochemistry results we found positive HIF-1 a and ANXA1 were stained in brown in cytoplasm with or without nuclear staining (Fig. 1A). Positive HIF-1 a expression was observed in approximately $82.9 \%$ of the GC versus ANXA1 35.5\% (Fig. 1A, Table 1). The result of immunoreactivity scores (IRS) of HIF-1a and ANXA1 staining available from the GC tissue microarray were consistent with the findings of immunohistochemistry analysis (Fig. 1B).

For verification of HIF-1a and ANXA1 expressions in GC tissues, we next collected 10 pairs of frozen GC samples and none cancer gastric tissues for further Western blot analysis. As shown in Fig. 1C, the expression of HIF-1a was higher versus ANXA1 in same GC tissues which indicated that HIF-1a and ANXA1 may be negative regulatory relationship.

\section{Increased HIF-1a correlates with clinicopathological parameters in GC patients}

Assessment of the association between HIF-1a expression and the clinicopathological data is presented in Table 2. Our data showed that increased expression of HIF-1a was significantly correlated with the diameter of $\mathrm{GC}(P=0.026)$. We also found increased HIF-1 a expression was significantly correlated with lymph node metastasis $(P=0.011)$. Whereas, we did not find significant correlation between HIF-1a with other clinicopathologic variables, including age, gender, TNM stage, tumor differentiation and hematogenous metastasis.

However, we did not find significant correlation between ANXA1 with any clinicopathologic variables. Assessment of the association between ANXA1 expression and the clinicopathological data is presented in supplementary Table 1.

\section{HIF-1a serves as a potential prognostic indicator for GC patients}

To further study whether the expressions of HIF-1 $a$ and ANXA1 in GC patients correlate with the prognosis of GC patients, Samples with the IRS of 0-4 were classified as low-expressing, and samples with the IRS of 5-12 were identified as having high HIF-1 a and ANXA1 expressions. Our results revealed that high HIF- 
1a expression was correlated with worse overall survival in GC patients $(P=0.000$, Fig. 1D). However, there was no correlation of ANXA1 with the prognosis of GC patients $(P=0.107$, Fig. 1D).

Furthermore, to determine whether the expression of HIF-1a was an independent prognostic marker for GC patients, we analyzed HIF-1a, gender, age, tumour differentiation, tumor diameter, lymph node metastasis, TNM stage and hematogenous metastasis by univariate and multivariate Cox regression analysis. Univariate analysis (Table 3 ) revealed that HIF-1 a, lymph node metastasis and TNM stage were correlated with survival of GC patients. In the multivariate analysis (Table 3), only HIF-1a remained as a significant independent prognostic factor of decreased survival of GC patients. Taken together, HIF-1a may serve as an independent prognostic factor in GC.

Table 3

Univariate and multivariate analysis of HIF-1a and clinicopathological factors predicting survival of GC patients

\begin{tabular}{|c|c|c|c|c|}
\hline \multirow[t]{2}{*}{ Variables } & \multicolumn{2}{|l|}{ Univariate analysis } & \multicolumn{2}{|l|}{ Multivariate analysis } \\
\hline & $\mathrm{HR}(95 \% \mathrm{Cl})$ & $p$-value & $\mathrm{HR}(95 \% \mathrm{Cl})$ & $p$-value \\
\hline HIF-1a & $3.660(1.838-7.288)$ & 0.000 & $2.750(1.341-5.640)$ & 0.006 \\
\hline Gender & $0.828(0.407-1.685)$ & 0.603 & - & - \\
\hline Age & $0.794(0.418-1.507)$ & 0.480 & - & - \\
\hline \multicolumn{5}{|c|}{ Tumor size (Diameter) } \\
\hline & $1.721(0.904-3.279)$ & 0.098 & - & - \\
\hline \multicolumn{5}{|c|}{ Lymph node metastasis } \\
\hline & $0.349(0.159-0.767)$ & 0.009 & $0.498(0.210-1.182)$ & 0.114 \\
\hline \multicolumn{5}{|c|}{ Tumour differentiation } \\
\hline & $0.888(0.446-1.767)$ & 0.735 & - & - \\
\hline \multicolumn{5}{|c|}{ Hematogenous Metastasis } \\
\hline & $0.597(0.248-1.435)$ & 0.249 & - & - \\
\hline TNM & $2.023(1.052-3.894)$ & 0.035 & $1.250(0.613-2.545)$ & 0.539 \\
\hline
\end{tabular}

\section{Relationship between expressions of HIF-1a and ANXA1 in GC tissues and GC patients}

We searched for the correlation between HIF-1 $a$ and ANXA1. We found that the expression of HIF-1a was negatively correlated with ANXA1 $(P=0.032)$ (Spearman's rank correlation test) (Table 4), which was consistent with WB result (Fig. 1C). 
Kaplan-Meier analysis was further applied to compare overall survival of GC patients according to combinations of HIF-1 $a$ and ANXA1 (Fig. 1D). Patients with low HIF-1a plus low ANXA1 had the longest survival of GC patients, while those with high HIF-1a plus high ANXA1 had worse survival.

\section{Hypoxia increases HIF-1a expression and promotes GC cell migration and invasion}

To explore the effect of hypoxia on GC cells, we detected the expressions of HIF-1a in GC cells and the migration and invasion of GC cells. The results showed that HIF-1a was increased after $6 \mathrm{~h}$ and $12 \mathrm{~h}$ after co-cultured with $\mathrm{CoCl} 2$ (Fig. 2A). As HIF-1a and HIF-2a expressions were induced under hypoxia in HepG2 cells [17], we also detected HIF-2a expression. There's no change of HIF-2a expression being detected under hypoxia in both HGC27 and MGC803 cells (Fig. 2A), we concluded that HIF-2a was not involved in our experiment.

We continued to examine the effects of hypoxia on the migration and invasion of GC cells via wound healing and transwell assays. The width of the scratches in the wound healing assay at $0 \mathrm{~h}, 6 \mathrm{~h}$ and $12 \mathrm{~h}$ (Fig. 2B) was analyzed. The result showed that the speeds of wound healing in HGC27 and MGC803 cells co-cultured with $\mathrm{CoCl} 2$ were faster than that in the control groups. In transwell filter assays, we found that hypoxia significantly enhanced the ability of HGC27 and MGC803 cells to migrate through transwell filter inserts (Fig. 3A). We further examined the effects of hypoxia on the invasion ability of GC cells. We found that hypoxia significantly enhanced the ability of HGC27 and MGC803 cells to invade through the transwell filter inserts (Fig. 3B).

\section{Hypoxia promotes the migration and invasion of GC cells through HIF1a/ANXA1/MMP-2 pathway}

To investigate the mechanisms that hypoxia regulates the migration and invasion of GC cells, we detected HIF1a, ANXA1, MMP-2, TIMP-2, MMP-9 and TIMP-1 protein expressions in HGC27 and MGC803 cells by western blot. Our data showed that HIF1a increased, ANXA1 decreased, MMP-2 increased and TIMP-2 downregulated by hypoxia in GC cells (Fig. 4A). We further detected the activity of MMP-2 by gelatin zymography and we found that the activity of MMP-2 was positively regulated by hypoxia (Fig. 4B). Therefore, we supposed that hypoxia could promote the migration and invasion of GC cells by regulating HIF1a/ANXA1/MMP-2 pathway.

To further validate our hypothesis, immunoprecipitation analysis clearly indicated interactions of HIF1a, ANXA1 and MMP-2 in both HGC27 and MGC803 cells (Fig. 4C). In addition to the immunoprecipitation analysis, we determined the subcellular localization of HIF1a, ANXA1 and MMP-2 via immunofluorescence staining. The result showed that proteins (HIF1a, ANXA1 and MMP-2) are all localized in the cytosol (Fig. 5A, 5B). In addition, after ANXA1 siRNA was transfected into GC cells (Fig. 5C), the expression of MMP-2 was upregulated (Fig. 5D, 5E). Our results confirmed that under hypoxia condition, HIF1a regulated the expression of MMP-2 through ANXA1. 
Furthermore, we examined whether the migration and invasion of GC cells was regulated by MMP-2 via blocking MMP-2 by the selective inhibitor of it: SB-3CT (292605-14-2, MedChemExpress, USA) (Fig. 6A). Migration (Fig. 6B, 7A) and invasion (Fig. 7B) abilities were inhibited by inhibiting MMP-2 in GC cells. Above all, our results confirmed that hypoxia promotes the migration and invasion of GC cells through HIF1a/ANXA1/MMP-2 pathway.

\section{Discussion}

Hypoxia is defined as the increase in consumption of oxygen or reduction of oxygen relatively to the supply in cells, tissues and organs. Solid tumor cells survived in a microenvironment of hypoxia known as hypoxic adaptation. HIF1 a plays the critical role in tumor hypoxic adaptation and it may mediate series of downstream signaling pathways involved in tumor metastasis including epithelial to mesenchymal transition [18], invasion the extracellular matrix and the secondary growth of metastases [19]. $\mathrm{CoCl}_{2}$ has been demonstrated to enhance HIF1a expression under its induced tumor hypoxia environment in vivo $[18,20]$. In this study, hypoxia condition was induced by $\mathrm{CoCl}_{2}$ and our study showed the expression of HIF1a was strongly increased. Furthermore, our results showed that hypoxia promoted the migration and invasion of GC cells.

An important protein of one of the downstream pathways under hypoxia environment regulated by increased HIF1a is ANXA1 [11]. Growing evidences have suggested that ANXA1 contributes to the progress of tumor metastasis of several human tumors. ANXA1 has been reported regulating miR-562 and miR-26b through targeting NF-kb and angiogenesis to control metastasis of breast cancer [21]. Recent sports have shown that ANXA1 functions as regulator of MMPs in tumor metastasis. ANXA1 functions as regulator of MMP-9 expression and activates the NF-KB signaling pathway involved in invasion of breast cancer cells [22]. Chang Mei Hu, et al have reported that miR-196a modulates migration and invasion of esophageal squamous carcinoma cells through ANXA1 negatively regulating COX2, MMP-2, Snail and positively regulating E-cadherin [23].Our previous study has showed that ANXA1 knockdown inhibits cell proliferation by inducing $\mathrm{G} 1$ phase cell cycle arrest and increases migration and invasion of pancreatic ductal adenocarcinoma (PDAC) cells through up-regulating MMP-9 expression and activity [16]. Our hypothesis was that HIF1 a play crucial role in migration and invasion of GC cells under hypoxia condition through regulating ANXA1/MMP pathway.

In our present study, our data showed that under hypoxia condition, the expression of HIF1a increased, ANXA1 decreased, MMP-2 increased and TIMP-2 downregulated in HGC27 and MGC803 cells. We continuously detected the activity of MMP-2 by gelatin zymography and we found that the activity of MMP-2 was positively regulated by hypoxia. Furthermore, our immunoprecipitation and immunofluorescence results illustrated the interaction among HIF1a, ANXA1 and MMP-2. In addition, under hypoxia condition when knock down ANXA1 (siANXA1), as we expected, the expression of MMP-2 significantly increased in HGC27 and MGC803 cells. Together, these data support that HIF1a regulated MMP-2 through ANXA1. 
As is well known, MMPs contribute to cell migration and invasion during the early stages of malignant tumor through regulating the degradation of extracellular matrix (ECM) [24]. Among MMPs, MMP-2 and MMP-9 are type IV collagenases and can degrade specifically the basement membrane of ECM [25]. The expressions and activities of MMPs are regulated by multiple factors, among the factors, tissue inhibitors of matrix metalloproteinases (TIMPs) are the key precise factors [26]. The activities of MMP-2 and MMP9 are regulated respectively by TIMP-2 and TIMP-1. Our data showed that under hypoxia condition, the expression and activity of MMP-2 increased, the expression of TIMP-2 decreased. However, there's no change of the expression of MMP-9 and TIMP-1. Furthermore, the migration and invasion of GC cells under hypoxia condition were both inhibited by the inhibitor of MMP-2. Together, these data support that the migration and invasion of GC cells under hypoxia conditions were regulated through HIF1a/ANXA1/MMP-2 pathway.

\section{Conclusion}

In summary, based on our findings, we conclude that increased HIF-1 a expression was correlated with lymph node metastasis and poor survival rate of GC patient. HIF-1a may serve as an independent prognostic factor in GC and the combination of HIF-1a and ANXA1 might serve as a useful prognostic marker in GC. Furthermore, we have confirmed that HIF-1a can promote GC migration and invasion HIF-1a could promote GC migration and invasion through HIF1a/ANXA1/MMP-2 pathway. Our results support the potency of HIF-1a as novel biological marker for GC and its migration and invasion, thus may provide new insight for both diagnosis and cellular therapy of GC.

\section{Abbreviations}

GC: gastric cancer; HIF: hypoxia inducible factor; ANXA1: annexin A1; TME: tumor microenviroment; MMP-2: matrix metal proteinase 2; MMP-9: matrix metal proteinase 9; TIMP-2: tissue inhibitor of metalloproteinase 2; TIMP-1: tissue inhibitor of metalloproteinase 1; PDAC: pancreatic ductal adenocarcinoma; ECM: extracellular matrix; TIMPs: tissue inhibitors of matrix metalloproteinases.

\section{Declarations}

\section{Authors' contributions}

Qing-Hua Liu designed the study and performed the experiments; Lei Xia performed the experiments and prepared the figures; Chen-Chen Lu performed the experiments; Ying Liu designed the study and contributed to drafting the manuscript. All authors read and approved the final manuscript.

\section{Acknowledgements}

Not applicable.

\section{Competing interests}


The authors have declared that no competing interest exists.

\section{Availability of data and materials}

The datasets used and/or analyzed during the current study are available from the corresponding author on reasonable request.

\section{Consent for publication}

Not applicable.

\section{Ethics approval and consent to participate}

This study was approved by the Human Ethical Committee, the Institutional Review Boards of Affiliated Hospital of Xuzhou Medical University, and written informed consents were obtained from all patients.

\section{Funding}

This study was funded by grants from the national Natural Science Foundation of China (NO. 81502030)

\section{References}

1. Ferlay J, Soerjomataram I, Dikshit R, Eser S, Mathers C, Rebelo M, Parkin DM, Forman D, Bray F. Cancer incidence and mortality worldwide: sources, methods and major patterns in GLOBOCAN 2012. International journal of cancer Journal international du cancer. 2015;136(5):E359-86.

2. Chen W, Zheng R, Baade PD, Zhang S, Zeng H, Bray F, Jemal A, Yu XQ, He J. Cancer statistics in China, 2015. Cancer J Clin. 2016;66(2):115-32.

3. Zurleni T, Gjoni E, Altomare M, Rausei S. Conversion surgery for gastric cancer patients: A review. World J Gastrointest Oncol. 2018;10(11):398-409.

4. JH L, KK C, C Y, LH T, VE S. SS Y: - Lauren Histologic Type Is the Most Important Factor Associated With Pattern of. $D-0372354$ (- 1528 - 1140 (Electronic)):- 105-113.

5. Hirotsu $Y$, Hada M, Amemiya K, Oyama T, Mochizuki H, Omata M. Multi-regional sequencing reveals clonal and polyclonal seeding from primary tumor to metastases in advanced gastric cancer. Journal of gastroenterology 2020.

6. Hanahan D, Coussens LM. Accessories to the crime: functions of cells recruited to the tumor microenvironment. Cancer cell. 2012;21(3):309-22.

7. W H-S SPKDKPDALF. D T, K Z: - Inhibition of kras-derived exosomes downregulates immunosuppressive BACH2/GATA-3. D - 8213295 (- 1950-6007 (Electronic)):- 109461.

8. MC B. G DO, http://orcid.org I-O, D DB: - Targeting hypoxia in tumor: a new promising therapeutic strategy. $D-8308647(-1756-9966$ (Electronic)):- 8. 
9. Semenza GL. The hypoxic tumor microenvironment: A driving force for breast cancer progression. Biochim Biophys Acta. 2016;1863(3):382-91.

10. Vaupel P, Mayer A. Hypoxia-Driven Adenosine Accumulation: A Crucial Microenvironmental Factor Promoting Tumor Progression. Adv Exp Med Biol. 2016;876:177-83.

11. C NRFB, SJ G. L, J W, B K, N B, I DB, B S, H L et al: - Annexin A1 sustains tumor metabolism and cellular proliferation upon stable loss. $D-101532965$ (- 1949-2553 (Electronic)):- 6693-6710.

12. Emmanouilidi A, Paladin D, Greening DW, Falasca M. Oncogenic and Non-Malignant Pancreatic Exosome Cargo Reveal Distinct Expression of Oncogenic and Prognostic Factors Involved in Tumor Invasion and Metastasis. Proteomics. 2019;19(8):e1800158.

13. X G, https://orcid.org I-O, Y F, J L, Y Z: - Annexin 1-nuclear factor-kappaB-microRNA-26a regulatory pathway in the metastasis. $D$ - 101531441 (- 1759-7714 (Electronic)):- 665-675.

14. Belvedere R, Bizzarro V, Popolo A, Dal Piaz F, Vasaturo M, Picardi P, Parente L, Petrella A. Role of intracellular and extracellular annexin $\mathrm{A} 1$ in migration and invasion of human pancreatic carcinoma cells. BMC Cancer. 2014;14:961.

15. Liu QH, Yong HM, Zhuang QX, Zhang XP, Hou PF, Chen YS, Zhu MH, Bai J. Reduced expression of annexin $\mathrm{A} 1$ promotes gemcitabine and 5 -fluorouracil drug resistance of human pancreatic cancer. Investig New Drugs. 2020;38(2):350-9.

16. Liu QH, Shi ML, Bai J, Zheng JN. Identification of ANXA1 as a lymphatic metastasis and poor prognostic factor in pancreatic ductal adenocarcinoma. Asian Pacific journal of cancer prevention: APJCP. 2015;16(7):2719-24.

17. M EMIEGMIRJR, C R. M: - HIF1A gene transcription is dependent on a core promoter sequence encompassing. $D-0372516$ (- 0006-291X (Print)):- 534-540.

18. Y BF, L ZCSZS, C T. L, G Z: - Basil polysaccharide inhibits hypoxia-induced hepatocellular carcinoma metastasis. $D-7909578$ (- 1879-0003 (Electronic)):- 32-44.

19. Wong CC, Kai AK, Ng IO. The impact of hypoxia in hepatocellular carcinoma metastasis. Frontiers of medicine. 2014;8(1):33-41.

20. S C-P RO, A G-P, JL MBRJ. M, J G-G: - Inhibition of VEGF expression through blockade of Hif1alpha and STAT3 signalling. $D-0370635$ (- 1532-1827 (Electronic)):- 83-91.

21. G DA, VK YYY, WH P, JS LSAPB. W, G S, PM N et al: - Annexin-A1 regulates microRNA-26b* and microRNA-562 to directly target NF-kappaB. D - 101285081 (- 1932-6203 (Electronic)):- e114507.

22. Kang $\mathrm{H}, \mathrm{Ko} \mathrm{J}$, Jang SW. The role of annexin $\mathrm{A} 1$ in expression of matrix metalloproteinase-9 and invasion of breast cancer cells. Biochem Biophys Res Commun. 2012;423(1):188-94.

23. Hu C, Peng J, Lv L, Wang X, Zhou Y, Huo J, Liu D: miR-196a regulates the proliferation, invasion and migration of esophageal squamous carcinoma cells by targeting ANXA1. Oncology letters 2019, 17(6):5201-5209.

24. DeClerck YA, Mercurio AM, Stack MS, Chapman HA, Zutter MM, Muschel RJ, Raz A, Matrisian LM, Sloane BF, Noel A, et al. Proteases, extracellular matrix, and cancer: a workshop of the path B study 
section. Am J Pathol. 2004;164(4):1131-9.

25. Woessner HN. JF J: - Matrix metalloproteinases. D - 2985121r (- 0021-9258 (Print)):- 21491-21494.

26. Lenglet S, Mach F, Montecucco F. Role of matrix metalloproteinase-8 in atherosclerosis. Mediat Inflamm. 2013;2013:659282.

\section{Tables}

Table 4, mentioned on page 10, was omitted by the authors in this version of the paper

\section{Figures}



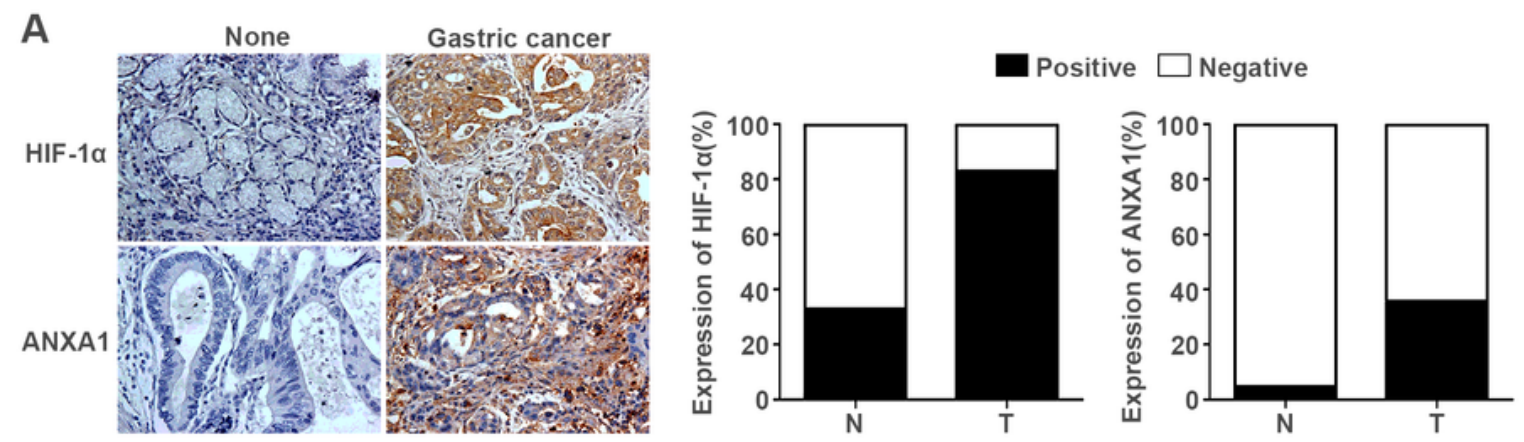

\section{B}

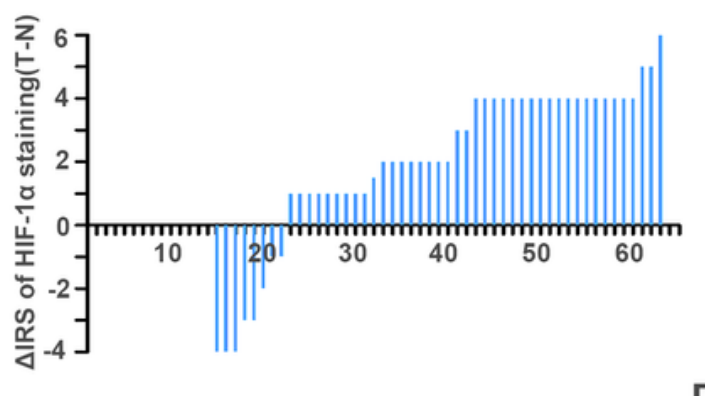

C
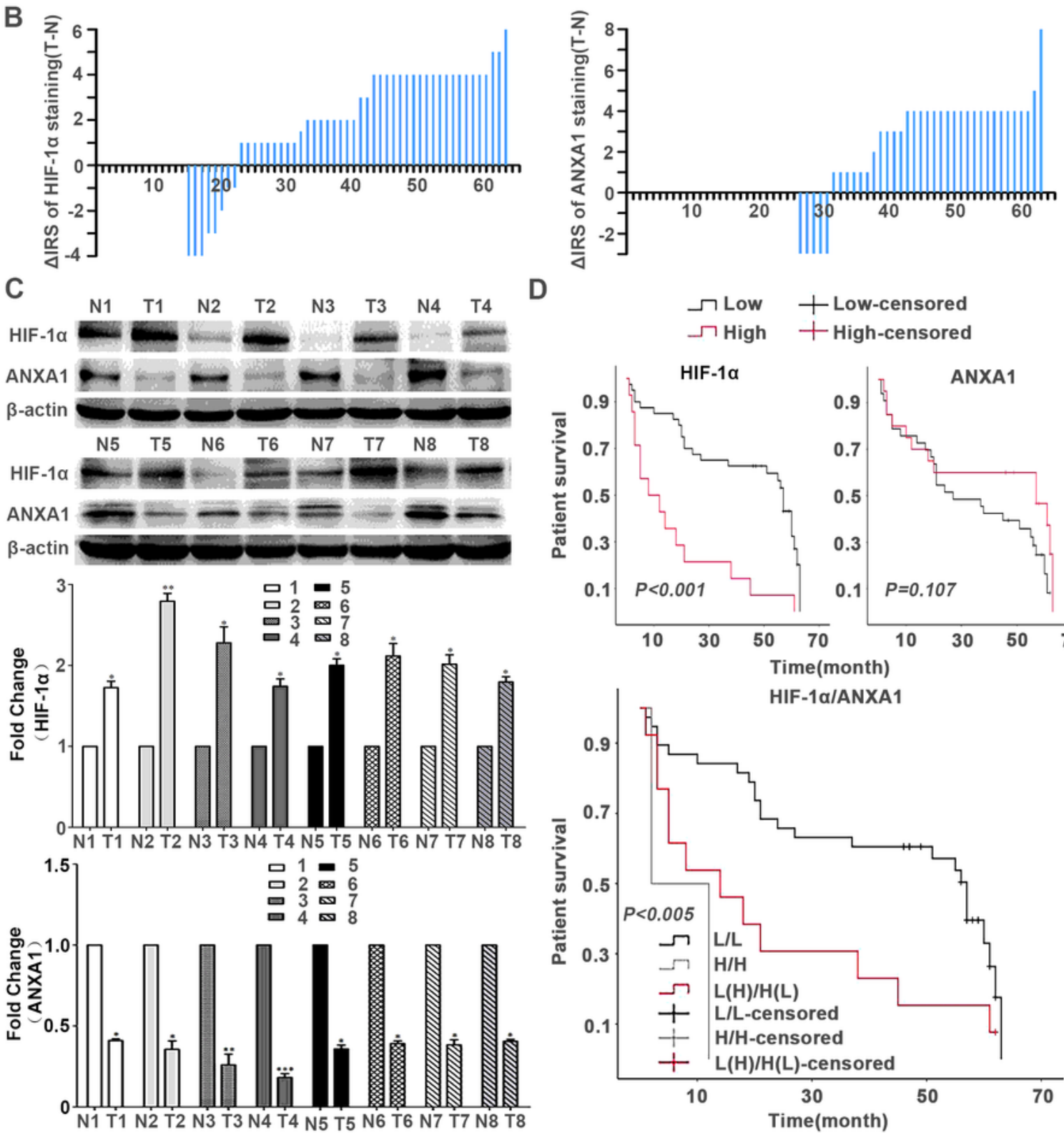

D
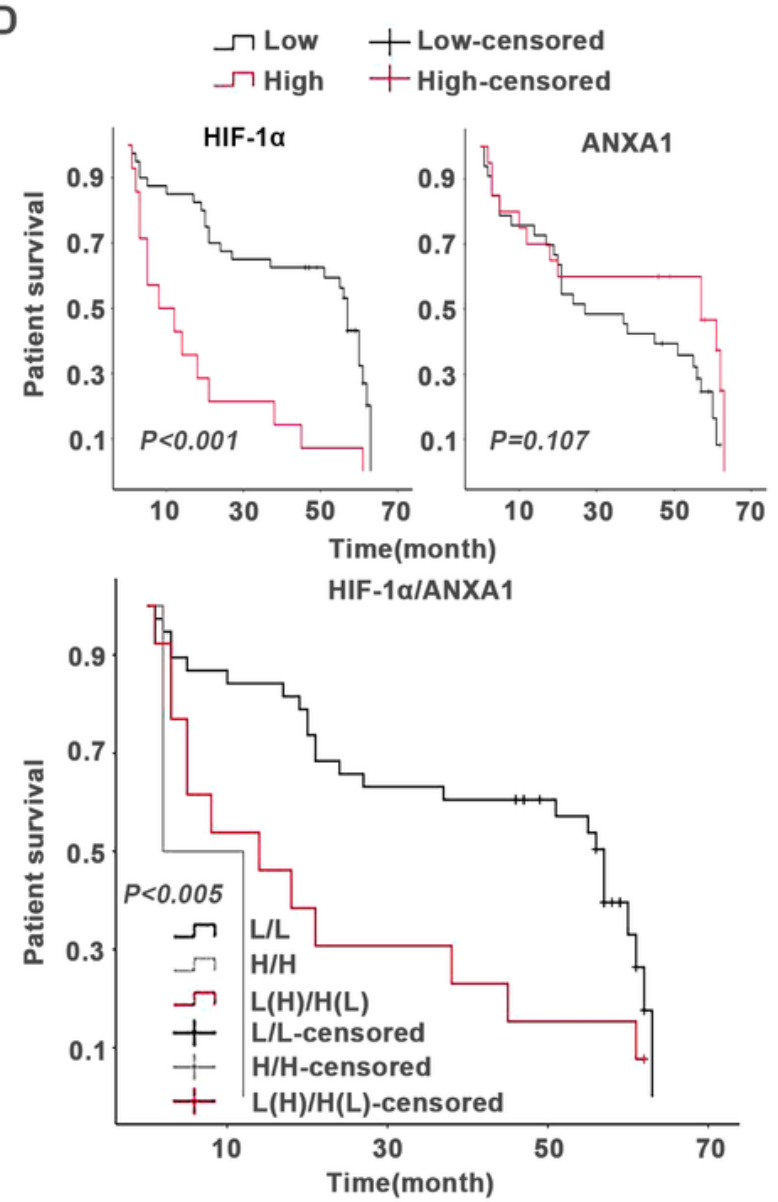

Figure 1

HIF-1a is increased in GC and associated with overall survival in GC patients. (A) Top panel, HIF-1a and ANXA1 immunohistochemical staining in GC and normal gastric tissues, $\times 400$. (B) Distributions of the difference HIF-1 $a$ and ANXA1 staining in in GC and normal gastric tissues were available from the 62 pairs of tissues. (C) The expression of HIF-1a was high versus low expression of ANXA1 determined by 
Western blot in 10 pairs of the same frozen gastric tissues. (D) High expression of HIF-1a correlated with worse overall survival. The combination of high HIF-1a plus high ANXA1 had worse overall survival.

A
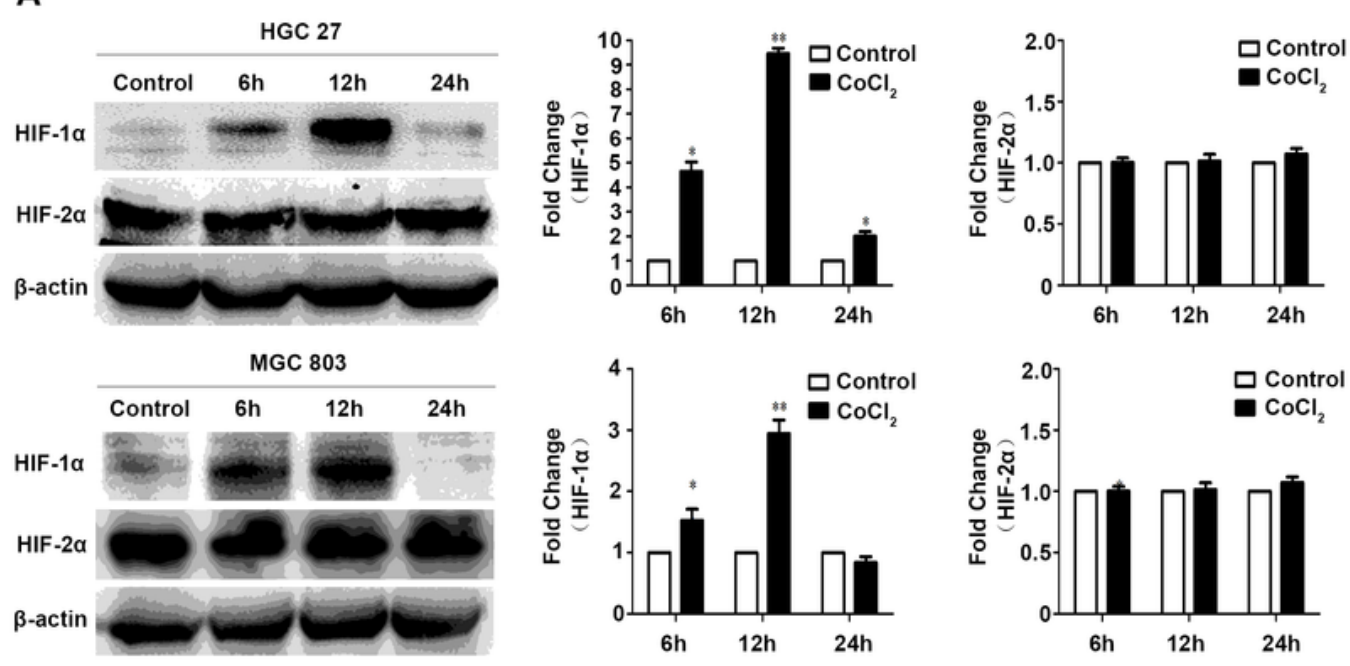

B

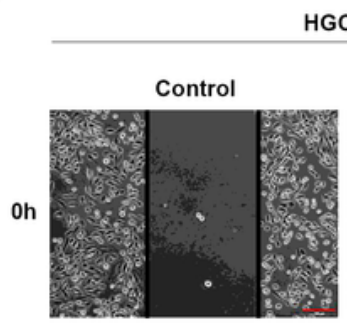

HGC 27

MGC 803
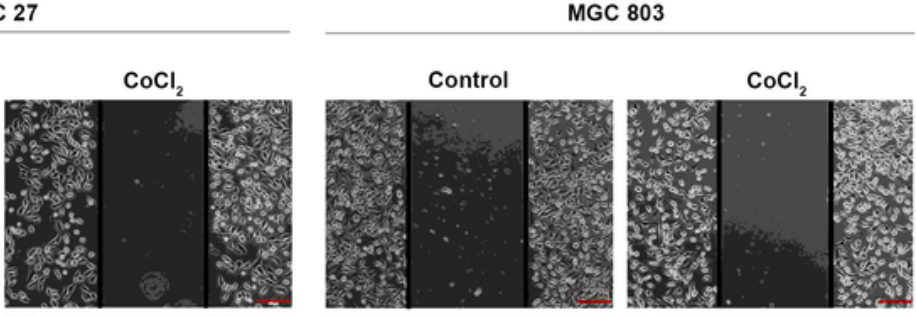

$6 \mathrm{~h}$
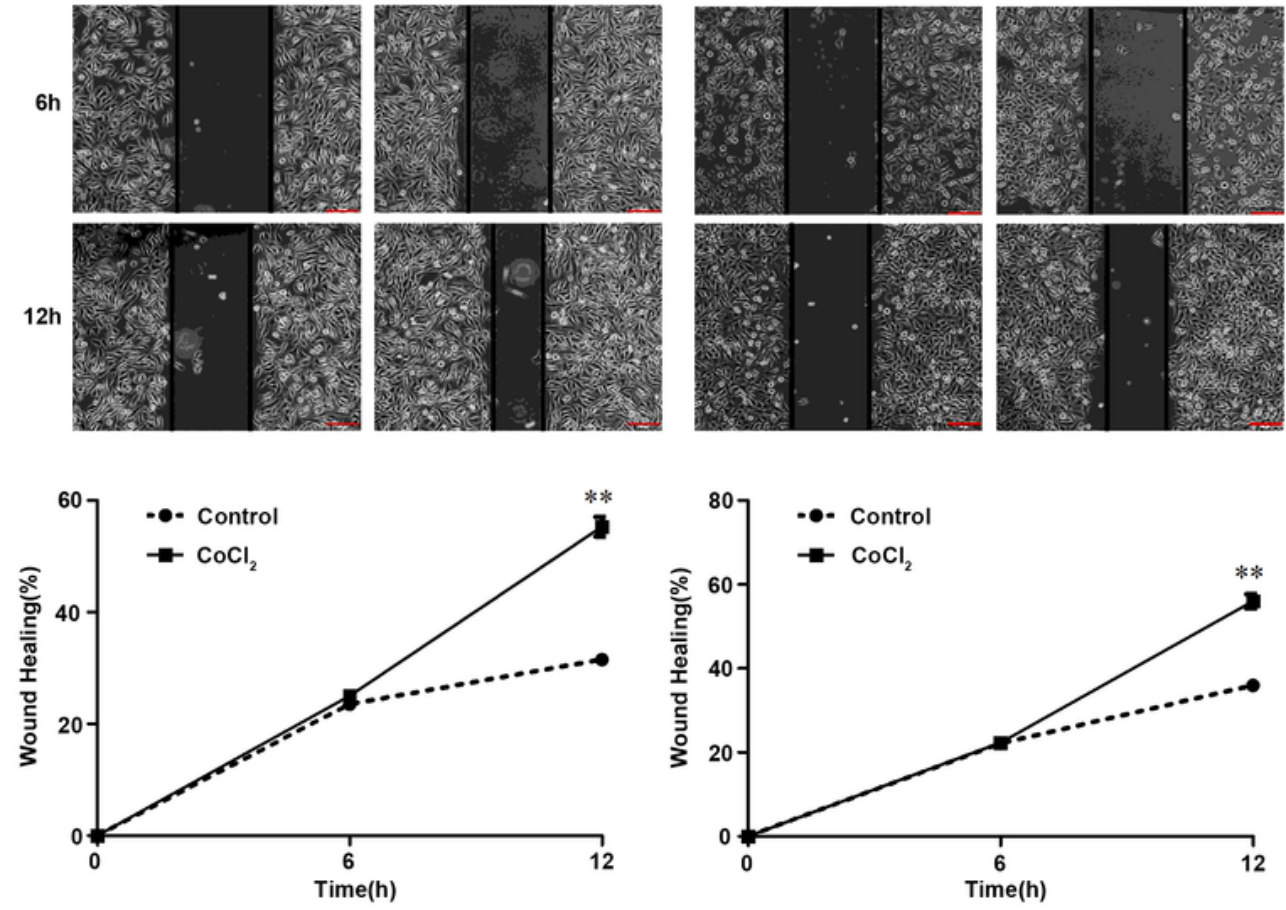

Figure 2

HIF-1a improves wound healing of GC cells. (A) The expression of HIF-1a was significantly increased in both HGC27 and MGC803 cells after $6 \mathrm{~h}$ and $12 \mathrm{~h}$ co-cultured with $\mathrm{CoCl} 2$. The expression of HIF-2a was no 
change after any hours both HGC27 and MGC803 cells co-cultured with CoCl2. (B) HIF-1a improved wound healing of both $\mathrm{HGC} 27$ and MGC803 cells after 12h co-cultured with $\mathrm{CoCl} 2$.

A

HGC 27
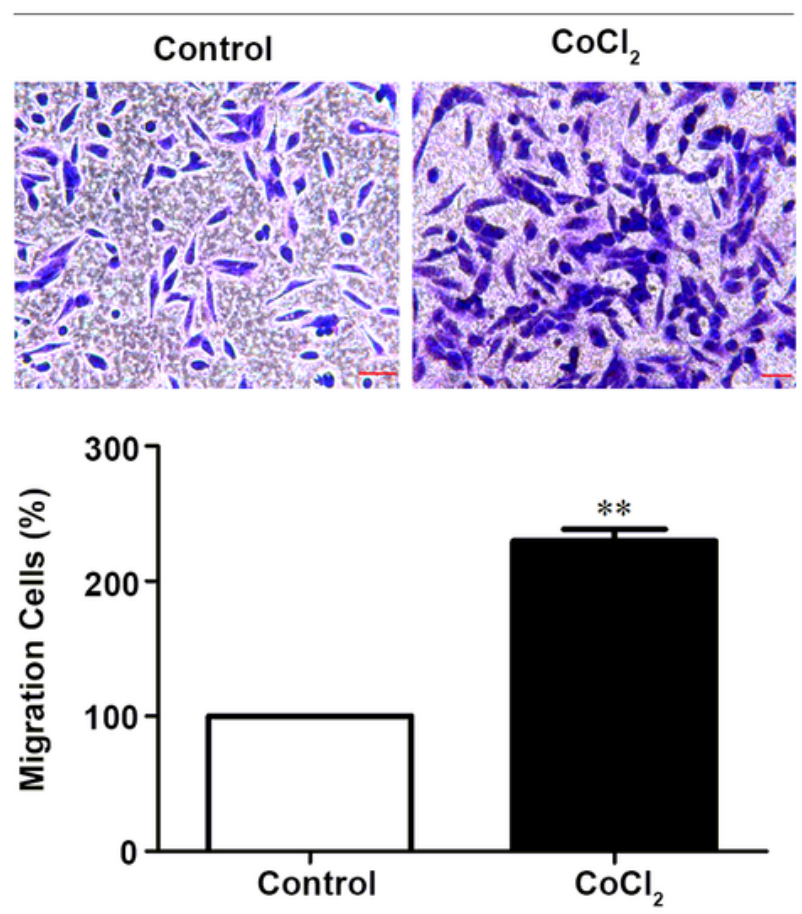

B
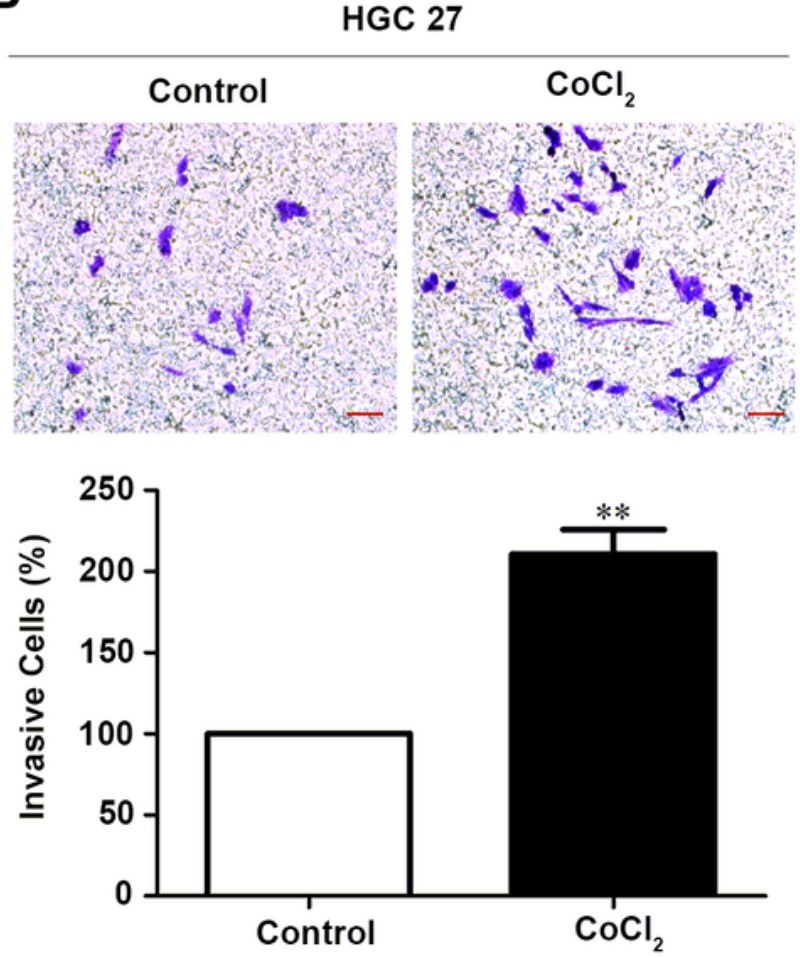

MGC 803
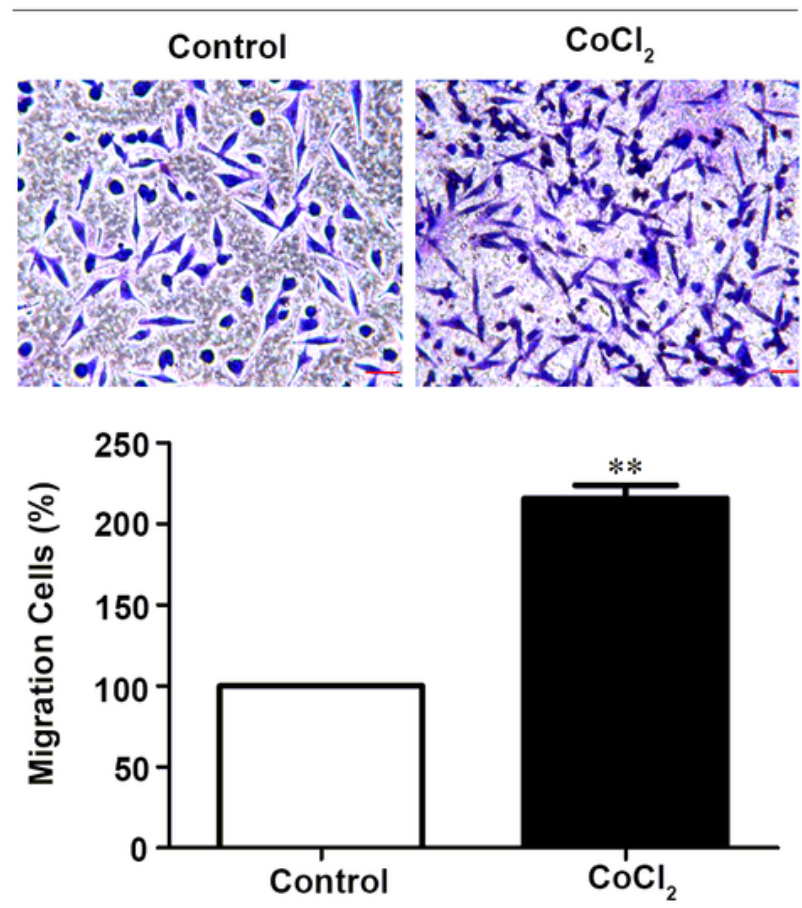

MGC 803
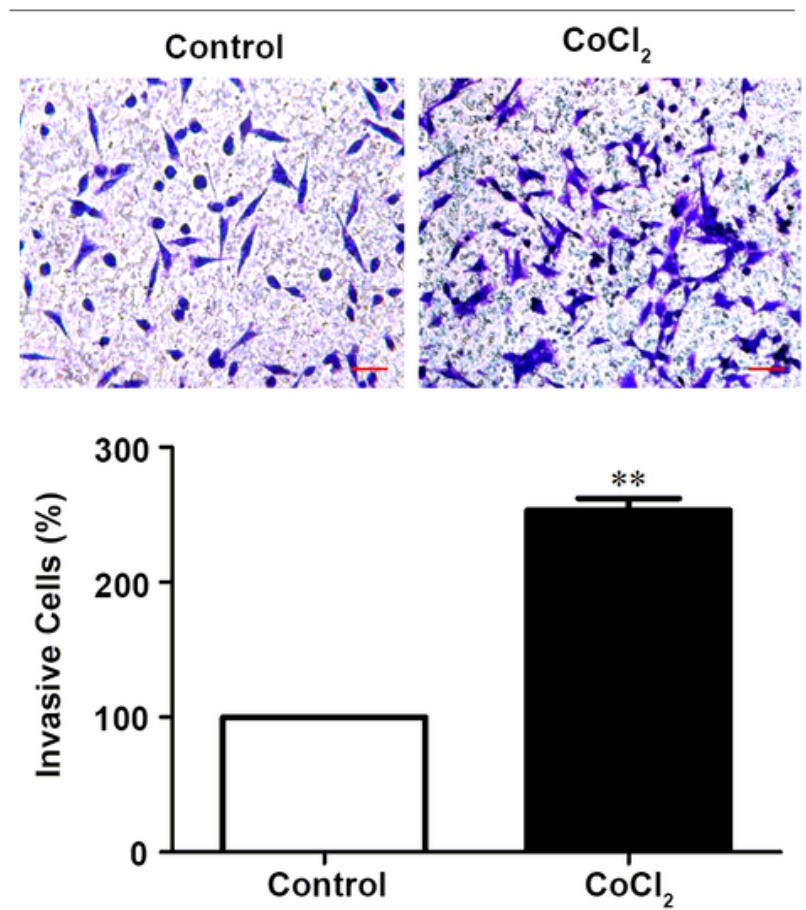

Figure 3

HIF-1a improves migration and invasion of GC cells. (A) HIF-1 a improved migration of both HGC27 and MGC803 cells. (B) HIF-1a improved invasion of both HGC27 and MGC803 cells. 
A
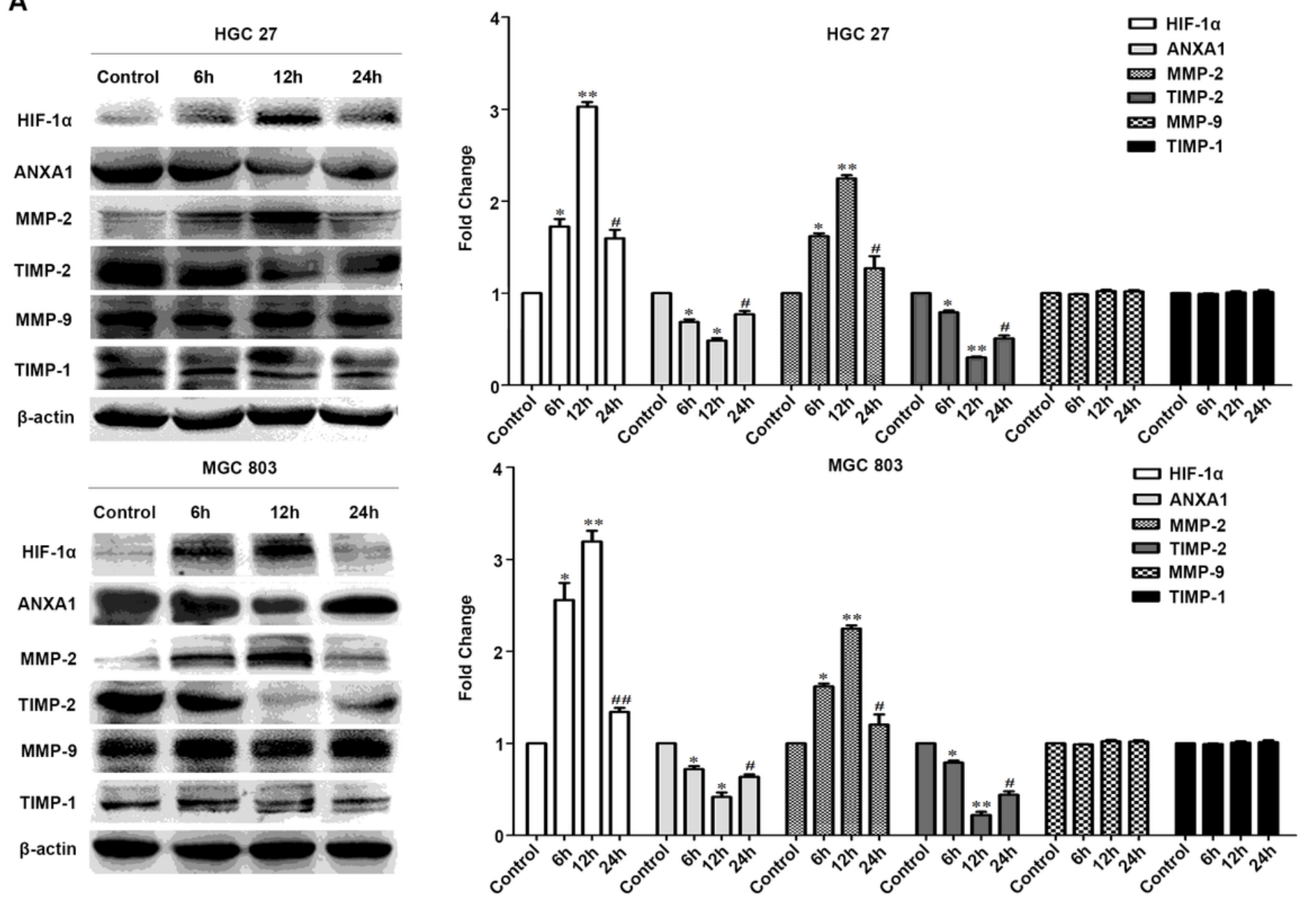

B

HGC 27

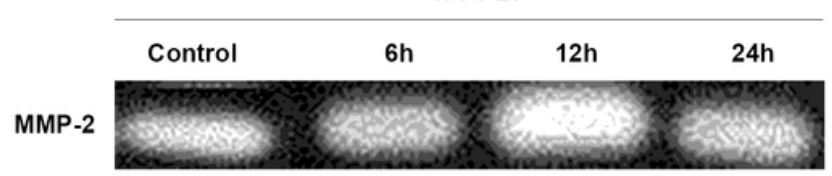

MGC 803

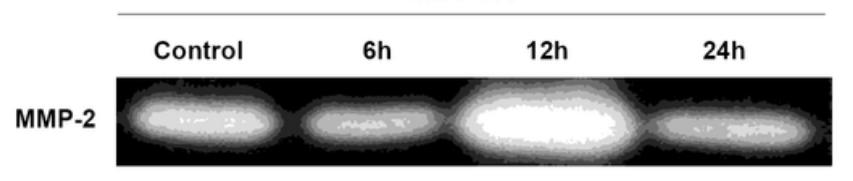

C
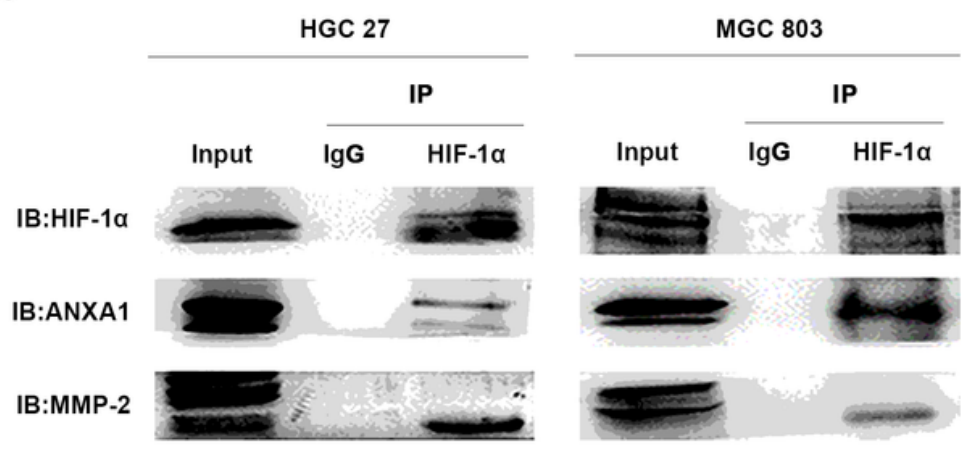

Figure 4

HIF-1a improves migration and invasion of GC cells through regulating ANXA1/MMP-2 signaling. (A) Western blot analysis of the relative protein levels of HIF-1a, ANXA1, MMP-2, TIMP-2, MMP-9 and TIMP-1 of both HGC27 and MGC803 cells after $6 \mathrm{~h}, 12 \mathrm{~h}$ and $24 \mathrm{~h}$ co-cultured with $\mathrm{CoCl} 2$. (B) Gelatin zymography analysis of the activity of MMP-2. (C) Immunoprecipitation analysis of the interactions of HIF1a, ANXA1 and MMP-2 in HGC27 and MGC803 cells. 
A

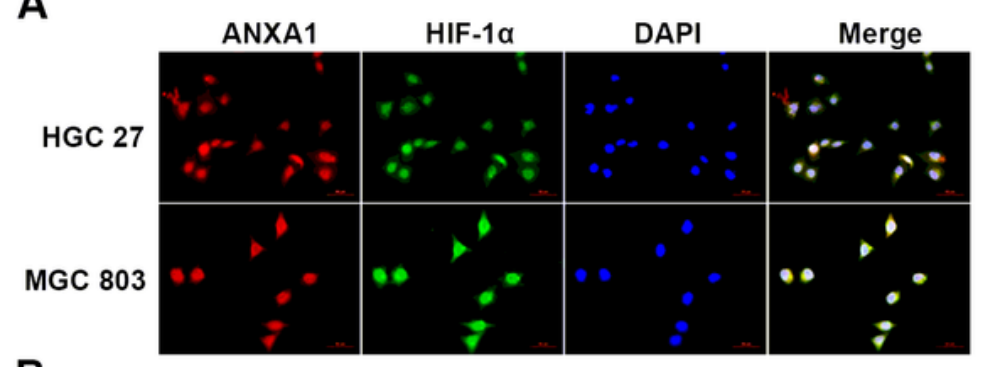

B

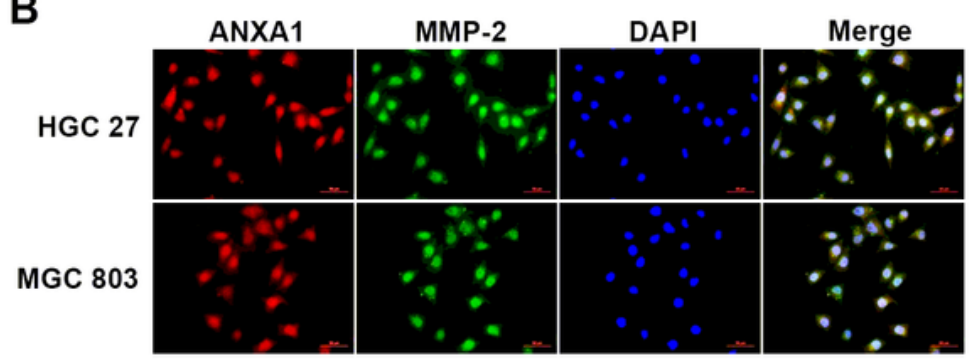

D

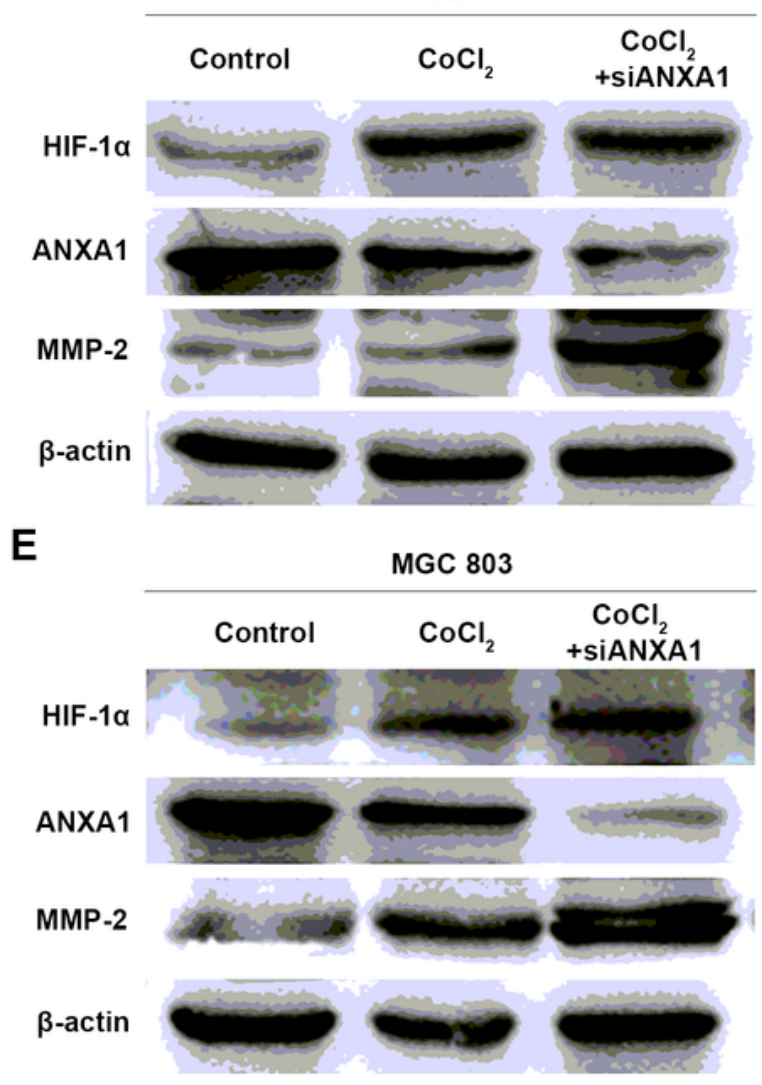

C
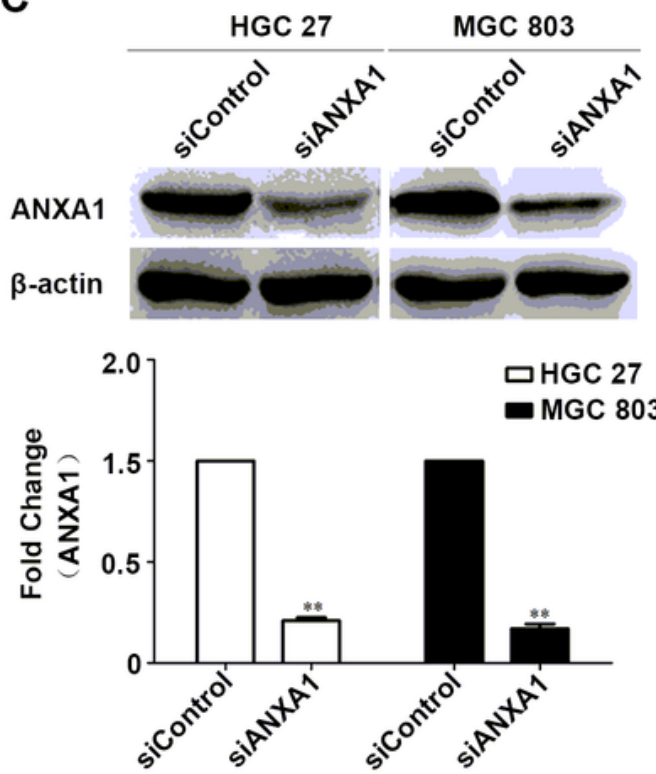

HGC 27

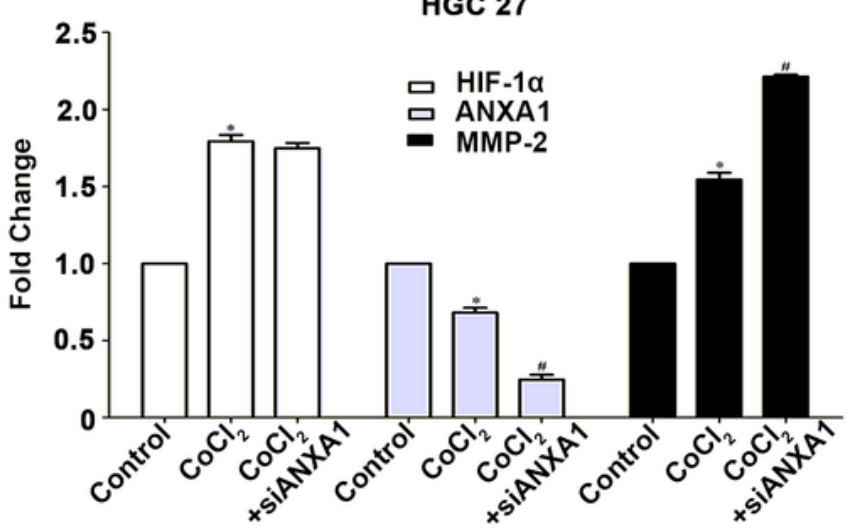

MGC 803

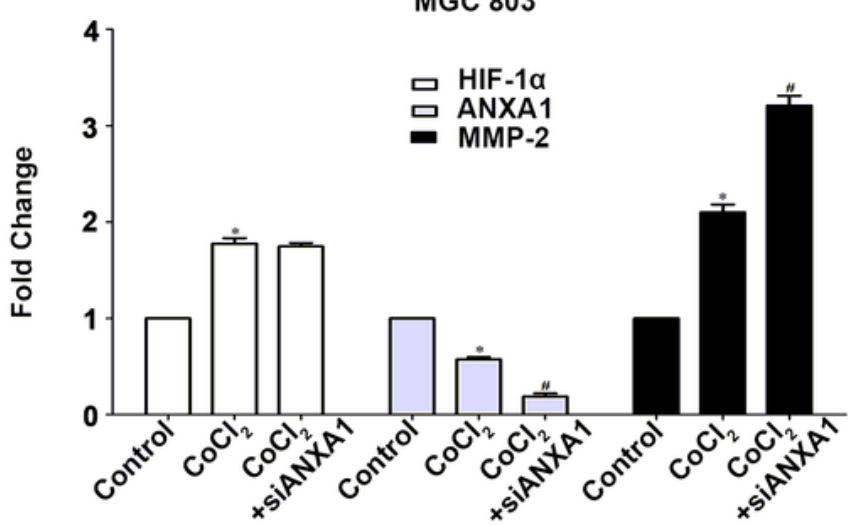

\section{Figure 5}

HIF1a regulated the expression of MMP-2 through ANXA1. Immunoflauorescence staining identified the subcellular localization of HIF1a, ANXA1 and MMP-2(A and B). The proteins (HIF1a, ANXA1 and MMP-2) are localized in the cytosol. NOTE: A. ANXA1 (red), HIF-1a (green), DAPI (blue); B. ANXA1 (red), MMP-2 (green), DAPI (blue). Original Magnification: $\times 40$. (C) ANXA1 siRNA was transfected into GC cells. (D, E) 
The expression of MMP-2 was upregulated after transfected ANXA1 siRNA in HGC27 and MGC803 cells under hypoxia condition.

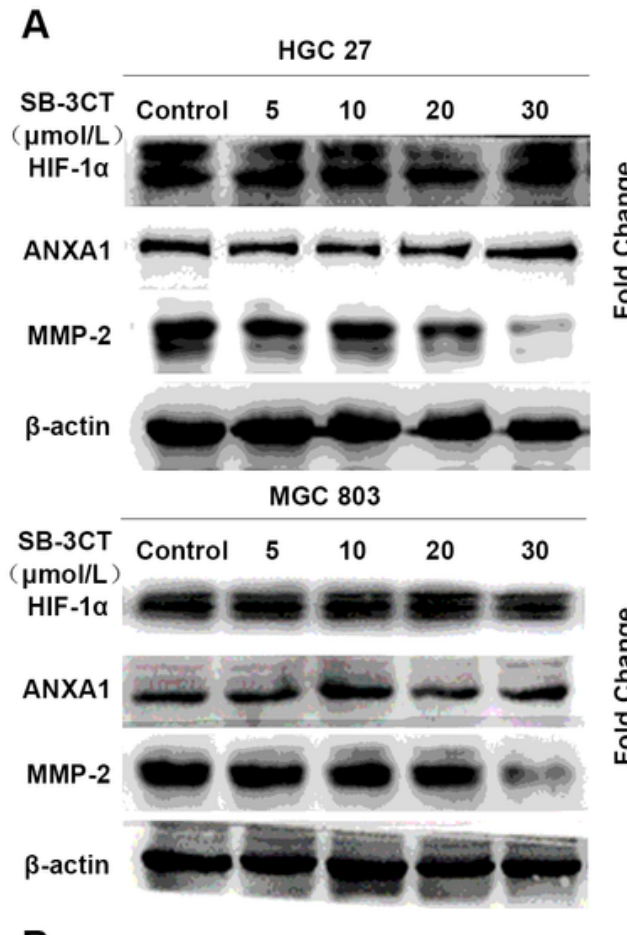

B

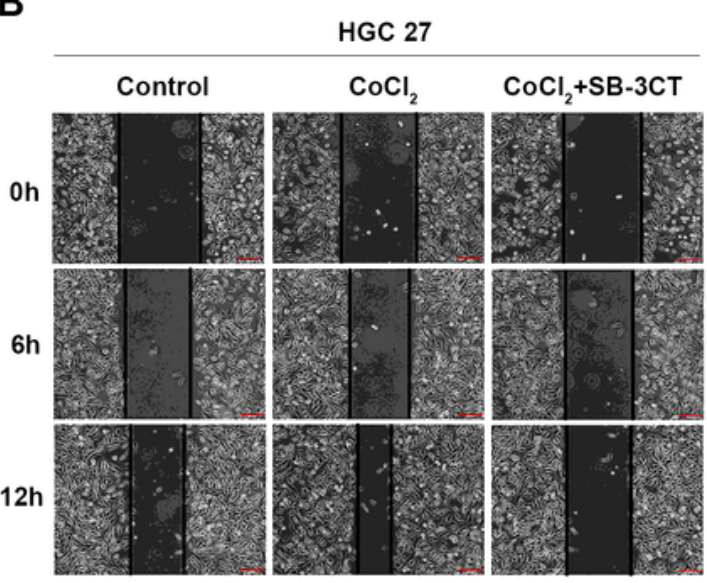

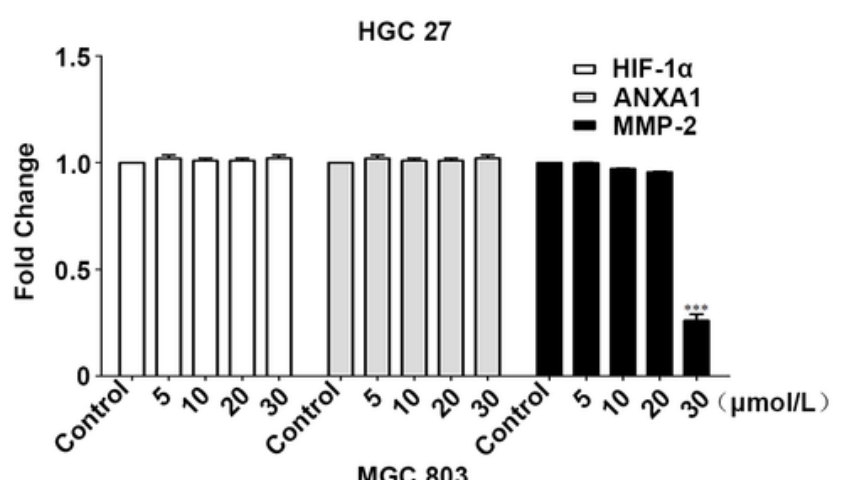

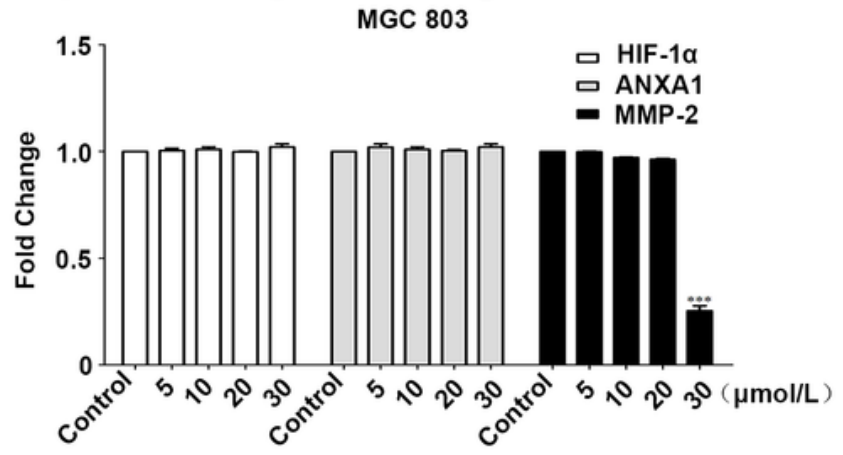

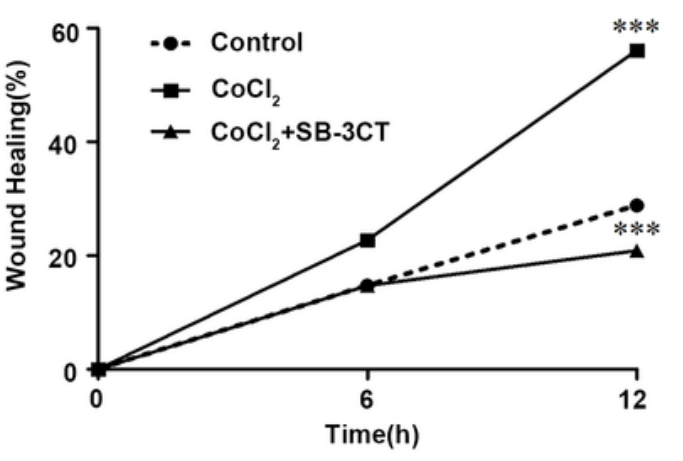
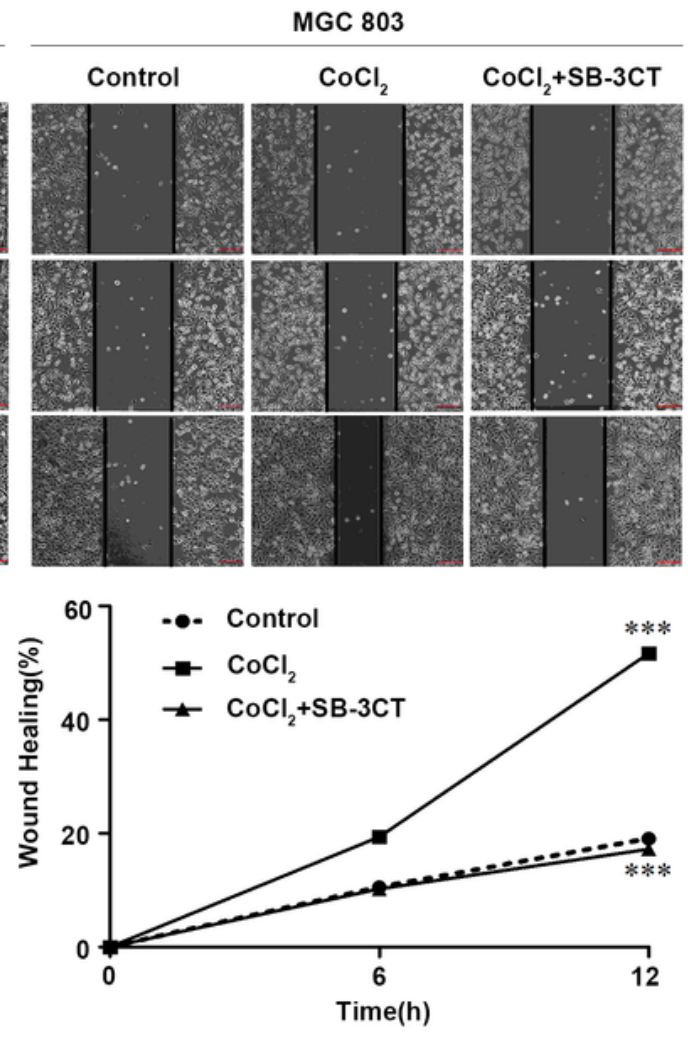

Figure 6

HIF1a regulated the migration and invasion of GC cells through HIF1a/ANXA1/MMP-2 pathway. (A) The

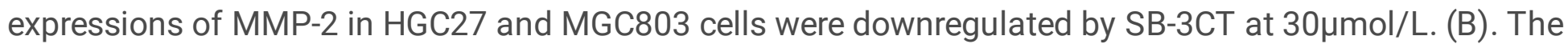
migration abilities were inhibited by SB-3CT in HGC27 and MGC803 cells at $12 \mathrm{~h}$. 

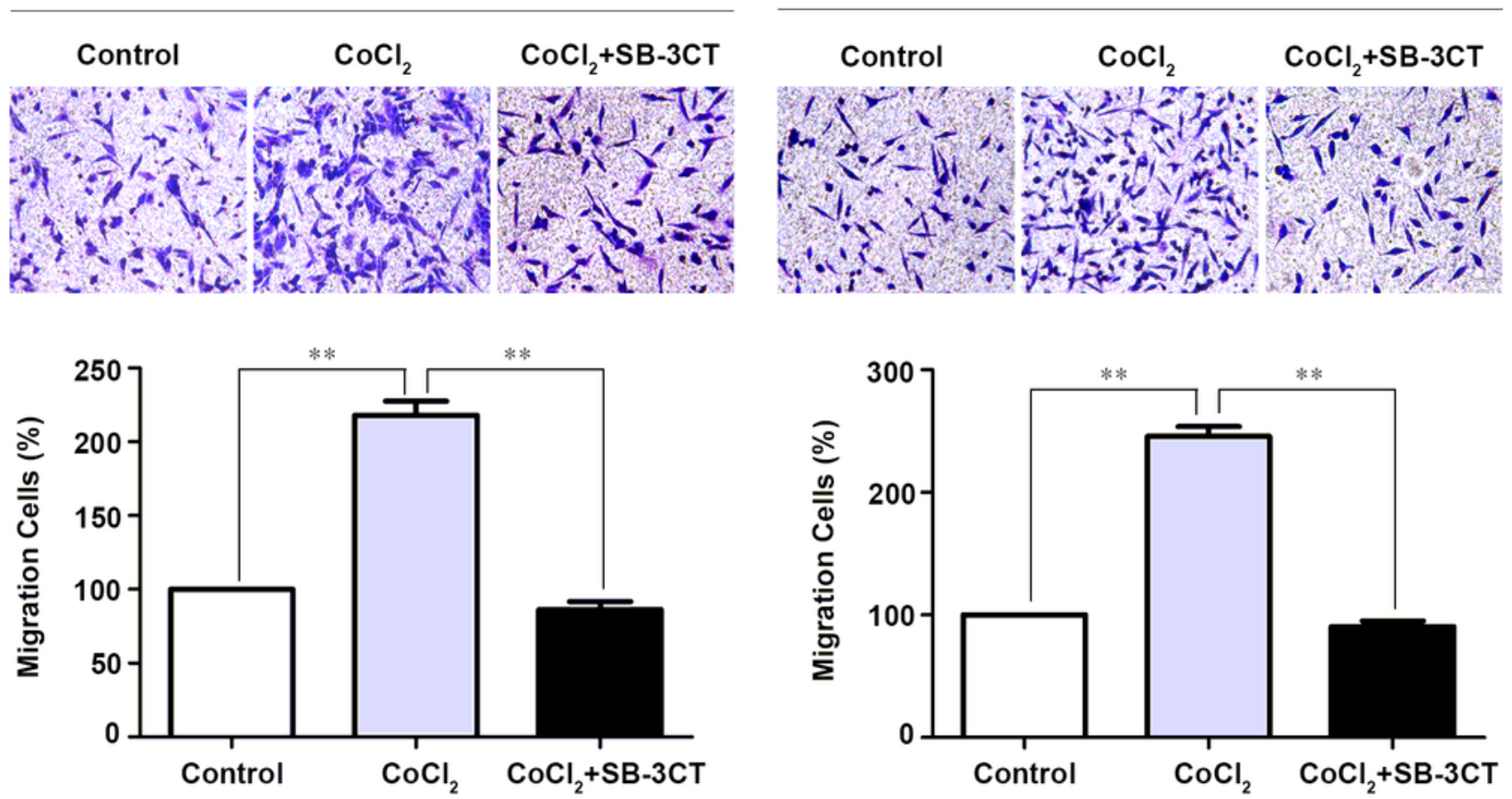

B

HGC 27

MGC 803
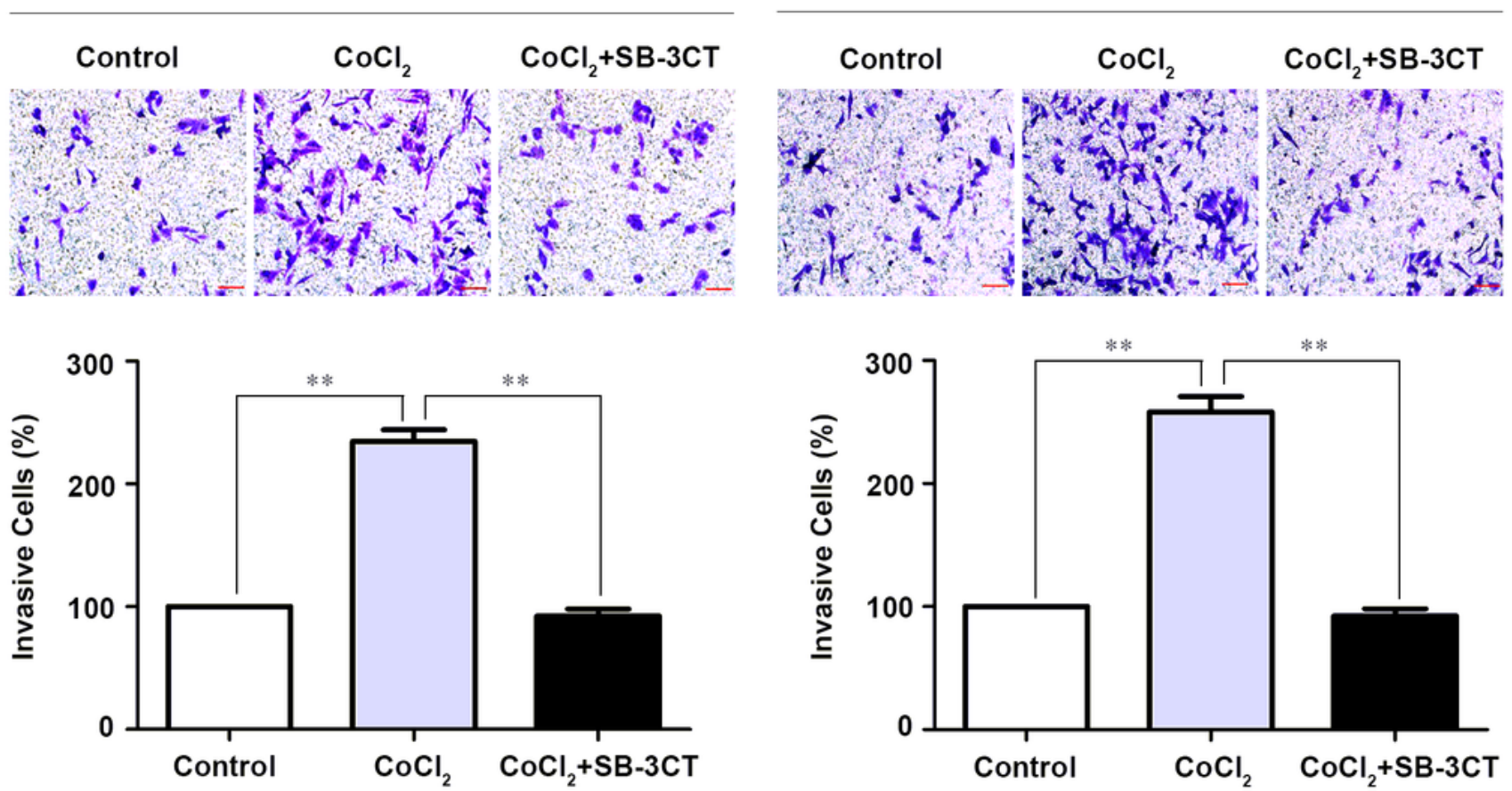

Figure 7

HIF1a regulated the migration and invasion of GC cells through HIF1a/ANXA1/MMP-2 pathway. (A) The migration abilities were inhibited by SB-3CT in HGC27 and MGC803 cells. (B) The invasion abilities were inhibited by SB-3CT in HGC27 and MGC803 cells.

\section{Supplementary Files}


This is a list of supplementary files associated with this preprint. Click to download.

- SupplementaryTable1.docx 